\title{
Comparative expression of Toll-like receptors and inflammatory cytokines in pigs infected with different virulent porcine reproductive and respiratory syndrome virus isolates
}

\author{
Lili Zhang, Jie Liu, Juan Bai, Xiaoye Wang, Yufeng Li and Ping Jiang ${ }^{*}$
}

\begin{abstract}
Background: Porcine reproductive and respiratory syndrome virus (PRRSV) is largely responsible for heavy economic losses in the swine industry worldwide because of its high mutation rate and subsequent emergence of virulent strains. However, the immunological and pathological responses of pigs to PRRSV strains with different virulence have not been completely elucidated.

Methods: Twenty-four piglets were divided into 4 groups ( $n=6$ each) and inoculated with highly pathogenic PRRSV isolate BB0907 (HP), low pathogenic PRRSV NT0801 (LP), LP derivative strain NT0801-F70 (LP-der), and DMEM medium (control), respectively. The changes in TLR2, 3, 7, and 8 gene expression and TNF- $\alpha, I L-1 \beta, I L-6, I F N-\gamma$, and IL-10 secretion were evaluated using real-time PCR and ELISA at 6, 9, and 15 days post inoculation (d.p.i.). The cytokine levels were evaluated in the supernatants of porcine alveolar macrophages (PAMs) and peripheral blood mononuclear cells (PBMCs) following stimulation with LTA, poly(l:C), CL097, and PRRSV individually.

Results: HP caused more severe clinical signs and pathological lesions in swine than LP and LP-der had almost no virulence compared with LP. The serum levels of IL-1 $\beta, I L-6, T N F-a$, and IFN- $\gamma$ were increased in HP-infected piglets, which were greater than in those infected with LP or LP-der. The mRNA levels of TLR3, 7, and 8 were significantly up-regulated in PAMs in HP-infected pigs compared to those in groups LP and LP-der. Furthermore, TNF- $a$ and IL-1 $\beta$ secretion in PAMs from group LP was statistically greater than those from the control group after stimulation with either poly(l:C) or CL097. Meanwhile, TNF-a, IL-1 $\beta$, and IL-6 levels in CL097-stimulated PBMCs from HP-infected pigs were markedly higher than those from the LP- and LP-der-infected groups.

Conclusions: We found that HP was a stronger inducer of TLR 3, 7, and 8 expression and IL-1 $\beta, I L-6$, TNF- $a$, and IFN- $\gamma$ production compared to LP and LP-der. HP enhanced production of TNF- $\alpha$, IL-1 $\beta$, and IL- 6 in PBMCs following CL097-stimulation more than LP and LP-der, whereas LP enhanced the secretion of TNF- $a$ and IL-1 $\beta$ in poly(I:C)and CL097-stimulated PAMs. Our data regarding cellular reactivity to different isolates should be useful in the development of more efficacious vaccines.
\end{abstract}

Keywords: PRRSV, Immunogenicity, Pathogenicity, TLRs, Cytokines

\footnotetext{
*Correspondence: jiangp@njau.edu.cn

Key Laboratory of Animal Diseases Diagnostic and Immunology, Ministry of Agriculture, College of Veterinary Medicine, Nanjing Agricultural University, Nanjing 210095, China
}

\section{Biomed Central}

(c) 2013 Zhang et al.; licensee BioMed Central Ltd. This is an Open Access article distributed under the terms of the Creative Commons Attribution License (http://creativecommons.org/licenses/by/2.0), which permits unrestricted use, distribution, and reproduction in any medium, provided the original work is properly cited. 


\section{Background}

Porcine reproductive and respiratory syndrome virus (PRRSV) is an enveloped positive-sense single-stranded RNA virus of the family Arteriviridae [1], which induces abortion in pregnant sows and gilts, and causes respiratory distress with increased susceptibility to secondary infections in piglets and growing pigs [2,3]. In China, highly pathogenic PRRSV (HP-PRRSV) has been isolated and identified as the causative agent of a large-scale PRRSV outbreak that has been on-going since 2006 and is characterized by a genomic marker with a 30-amino acid (aa) deletion in the nonstructural protein 2 (NSP2)coding region, compared to that in the PRRSV VR-2332 strain [4-6]. However, the pathological responses of different virulent PRRSV strains remain unknown.

Viral infections initiate a series of cellular events that lead to the generation of an antiviral state both in the infected cells and surrounding tissues [7]. Toll-like receptors (TLRs) are key components of the host innate recognition system, in which each TLR family detects distinct microbial pathogen-associated molecular patterns (PAMPs) and triggers the activation of specific signaling pathways, thereby inducing the transcription of inflammatory and/or anti-inflammatory cytokines [8]. For example, TLR3 recognizes double-stranded (ds) viral and synthetic RNA, such as polyriboinosinic polyribocytidylic acid (poly(I:C)) [9]. Once engaged, TLR3 triggers the activation of interferon regulatory factor 3 (IRF-3), a transcription factor with a critical role in the induction of type I interferon (INF) and nuclear factor kappa-light-chain-enhancer of activated $\mathrm{B}$ cells $(\mathrm{NF}-\mathrm{kB})$ through signaling processes that require the protein toll-interleukin-1 receptor domain-containing adaptor inducing IFN- $\beta$ (TRIF) $[10,11]$. Single-stranded (ss) RNA is the ligand recognized by TLR7 and 8 . These TLRs also trigger IRF-7-mediated type I IFN production upon activation, but unlike TLR3, the induction of IFN by TLR7 and 8 is coupled to the adaptor protein myeloid differentiation primary response gene 88 and not to TRIF. This recognition initiates signaling cascades that result in transcription factor activation ultimately leading to production of pro-inflammatory cytokines, chemokines, and/or anti-viral cytokines [12].

PRRSV is difficult to control due to its high mutation rate and the emergence of virulent strains [13-15], which may differently modulate immune responses based on cytokine expression [13-15] and PRRSV distribution in swine [13-15]. In the present study, three pathogenic PRRSV strains (highly pathogenic, HP; low pathogenic, LP; and low-derivative, LP-der) were analyzed and the modulation of TLR2, TLR3, TLR7, and TLR8 gene expression and tumor necrosis factor alpha (TNF- $\alpha$ ), interleukin (IL)-1 $\beta$, IL-6, IFN- $\gamma$, and IL-10 secretion were observed in vivo and in vitro. Our results showed that TLR3, TLR7, and TLR8 expression was correlated to
PRRSV virulence, which is consistent with cytokine levels in response to different infectious agents.

\section{Results}

\section{Clinical signs and body temperature changes}

After PRRSV challenge, all pigs infected with the HP PRRSV SY0608 strain had high fever $\left(\geq 41.0^{\circ} \mathrm{C}\right)$ and displayed a range of clinical signs, including inappetence, lethargy, rough hair coats, dyspnea, periocular edema, coughing, wheezing, and anhelation. Some pigs also showed eyelid edema, blue ears (cyanosis), muscle tremors, and diarrhea. Moreover, two of six pigs died at 9 days post-inoculation (d.p.i.), while pigs in the low pathogenic PRRSV NT0801 strain (LP) group showed a slight fluctuation in rectal temperatures and similar minor clinical signs at 15 d.p.i. Pigs in two groups, the NT0801 derivative strain NT0801-F70 (propagated in cultured African green monkey kidney-derived (MARC145 ) cells to 70 passages) (group LP-der) and Dulbecco's modified Eagle's medium (DMEM) (as the control group), had no clinical signs or significant increases in body temperature (Figure 1). The scores of clinical signs in the HP group were significantly higher than those in the other groups $(p<0.05)$ (Table 1$)$.

\section{PRRSV Viremia}

At 0, 3, 6, 9, and 15 d.p.i., pig blood samples were collected and serum PRRSV content was determined. As shown in Figure 2, pigs in group LP produced the highest levels of PRRSV viremia, which was significantly higher than those in groups LP-der and HP at 9 and 15 d.p.i. $(p<0.05)$ (Figure 2), while no viral content was detected in serum from the control pigs.

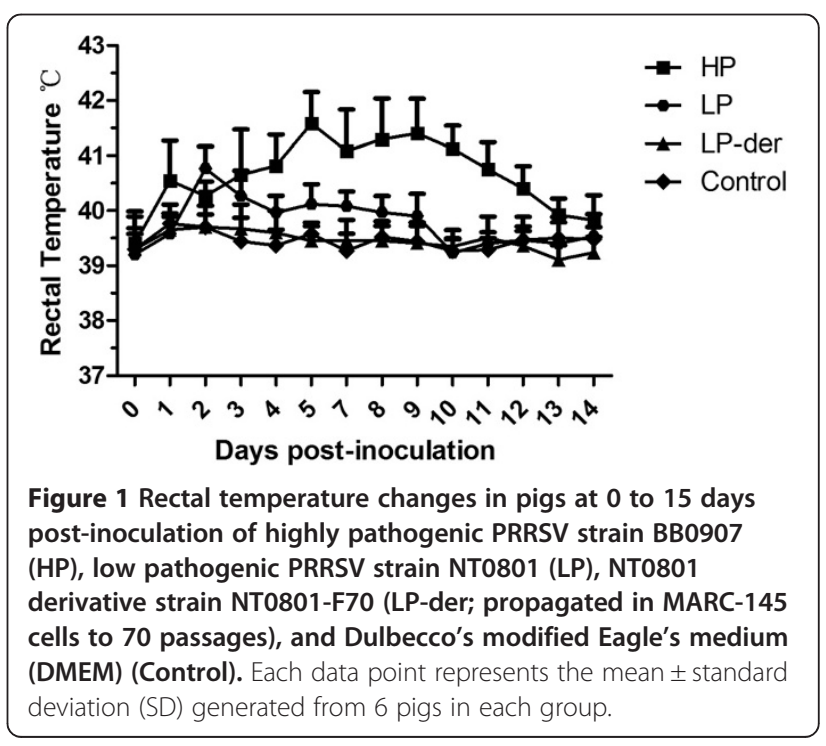


Table 1 The clinical sign scores of the pigs after challenge and their lung lesions recorded at 15 d.p.i. ${ }^{a}$

\begin{tabular}{|c|c|c|}
\hline Groups & Clinical signs scores $( \pm S D)^{b}$ & Lung lesions scores $( \pm S D)^{c}$ \\
\hline$\overline{\mathrm{HP}}$ & $10.4 \pm 1.22 \mathrm{~A}$ & $57.5 \pm 5.34 \mathrm{~A}$ \\
\hline LP & $7.2 \pm 0.92 \mathrm{~A}$ & $45.2 \pm 9.23 \mathrm{~A}$ \\
\hline LP-der & $1.8 \pm 0.35 B$ & $5.3 \pm 4.82 B$ \\
\hline Control & 0 & 0 \\
\hline
\end{tabular}

${ }^{a}$ Within each column, values followed by different letters (A, B) are significantly different $(p<0.05)$.

${ }^{b}$ Clinical sign scores are the sum of daily observations of behavior, respiration, and cough according to the severity of the illness.

'Evaluation of the percentage of the entire lung affected by pneumonia.

\section{Pathological examination}

At 15 d.p.i., all pigs were euthanized for pathological examinations, which showed that all members of the HPPRRSV group had diffuse tan consolidation of the lungs and occasional enlargement of the lymph nodes and spleen. As shown in Table 1, the lung lesion scores of HP-PRRSV-inoculated pigs were significantly higher than those in the other groups $(p<0.05)$, while mild lung lesions were observed in group LP, which were more severe than those in group LP-der.

On histological examination, the lung lesions in group HP-PRRSV pigs were characterized by thickened alveolar septa, infiltration with intensive lymphomononuclear cells, and hyperplasia of the bronchiolar epithelium (Figure 3A), whereas those in group LP-PRRSV exhibited mild interstitial pneumonia, which was lighter than that in group HP-PRRSV. The group LP-der pigs only exhibited light interstitial pneumonia (Figure 3), while there were no pathological changes in lung tissues from the control pigs.

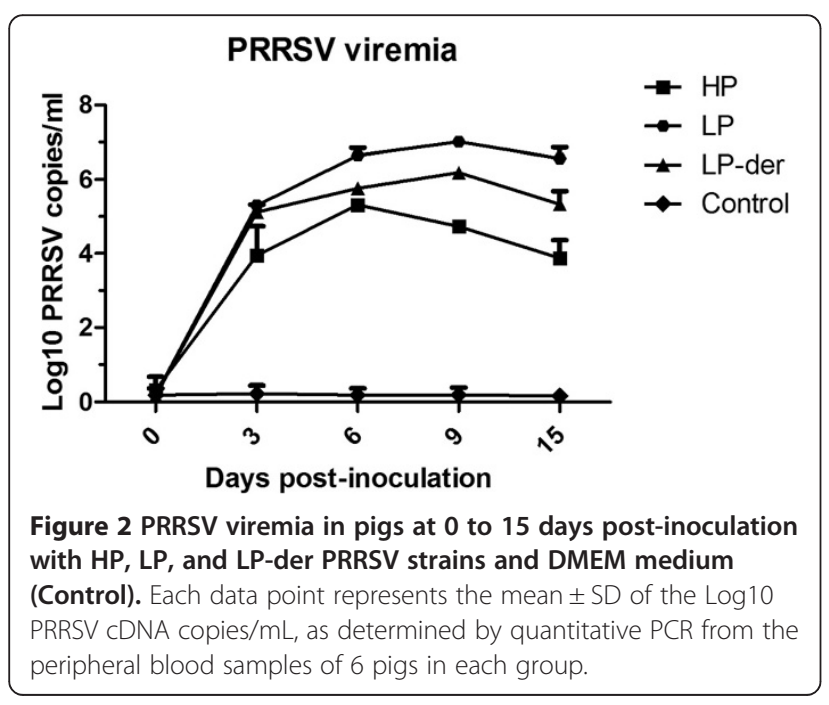

\section{Serum cytokine levels}

At $0,6,9$, and 15 d.p.i., blood samples were collected and the serum levels of TNF- $\alpha$, IL-1 $\beta$, IL-10, IL-6, and IFN- $\gamma$ were detected. As shown in Figure 4, the levels of TNF- $\alpha$, IL- $1 \beta$, and IL- 6 in the three viral groups rapidly increased at 6 d.p.i. and then gradually decreased until 15 d.p.i. In group HP, cytokine levels were significantly higher $(p<0.05)$ than those in groups LP and LP-der. Meanwhile, LP-PRRSV-inoculated pigs had relatively higher levels of TNF- $\alpha$, IL-1 $\beta$, and IL- 6 compared to those in group LP-der $(p<0.05)$. In the LP-der and control groups, there were no significant changes in those cytokine levels during the experiment (Figure 4).

Interestingly, IL-10 levels in group HP were lower than those in group LP at 9 and 15 d.p.i., even though they were increased in group HP at all time points compared with those in the control group. Meanwhile, IL-10 levels in group LP-der were lower than those in group LP, but higher than those in the control group (Figure 4D).

Although low levels of serum IFN- $\gamma$ were detected individually in PRRSV-infected pigs at 6 and 9 d.p.i., the levels in group HP pigs were significantly higher than those in the other groups at 15 d.p.i. $(p<0.05)$.

\section{TLR mRNA expression levels in PAMs and cerebral medulla}

The expression levels of TLR2, 3, 7, and 8 mRNA were examined individually by real-time polymerase chain reaction (PCR) in porcine alveolar macrophages (PAMs) and the cerebral medulla of pigs infected with the different PRRSV isolates. As shown in Figure 5A, the TLR3, 7, and 8 mRNA levels in PAMs of all PRRSV-infected pigs were higher than those of mock-infected pigs and the levels of TLR3 mRNA in PAMs from group HP-PRRSV were highest among all groups $(p<0.05)$. Meanwhile, the TLR2 mRNA expression level in group HP PAMs was also relatively higher than those of the other three groups $(p>0.05)$.

In the cerebral medulla, TLR3, 7, and 8 mRNA expression levels were significantly increased after HP-PRRSV challenge compared to those in group LP-der and mockinfected pigs $(p<0.05)$. TLR7 expression in group LP pigs was significantly higher than those in the LP-der $(p<0.05)$ and mock-infected groups $(p<0.01)$. However, there were no significant differences in TLR3, 7, and 8 mRNA levels in group LP-der compared with those of the control pigs (Figure 5B).

\section{Cytokine expression in PAMs and peripheral blood mononuclear cells (PBMCs) after PAMP stimulation} PBMCs and PAMs were collected from PRRSV- and mockinfected pigs and individually treated with lipoteichoic acid (LTA), poly(I:C), CL097, and PRRSV, or they remained untreated as controls. The culture supernatants were then collected to determine cytokine concentrations using an 

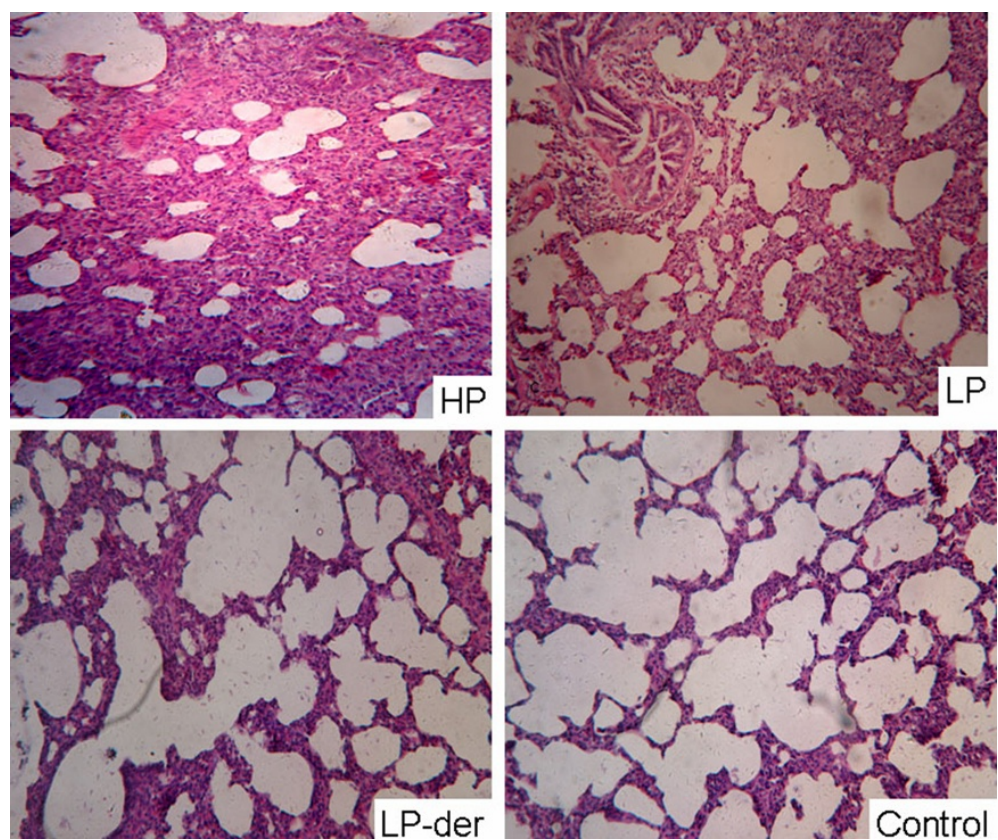

Figure 3 Pathological examination of lung tissues of pigs from groups HP, LP, LP-der PRRSV and the control group at 15 d.p.i. The tissues were stained with hematoxylin and eosin (magnification, x200).

enzyme-linked immunosorbent assay (ELISA). As shown in Figure 6, TNF- $\alpha$ levels in PAMs from all groups were significantly increased after being stimulated individually with poly(I:C), CL097, or PRRSV $(p<0.05)$ compared with unstimulated PAMs. The TNF- $\alpha$ concentrations in poly(I:C)-, CL097-, and PRRSV-treated PAMs from LP- and LP-der -PRRSV-infected pigs were significantly higher than those in HP- and mock-infected pigs $(p<0.05)$ (Figure 6A). Notably, CL097 and poly(I:C) significantly up-regulated IL-1 $\beta$ expression in PAMs from group LP compared to those from HP- and mock-infected pigs $(p<0.05)$ (Figure 6B). IL6 levels were significantly increased in CL097- and PRRSVtreated PAMs from groups HP and LP compared to those in group LP-der and the controls $(p<0.05)$ (Figure 6C).

Meanwhile, the TNF- $\alpha$, IL-1 $\beta$, and IL-6 levels in CL097-stimulated PBMCs from all groups were significantly higher than those in the untreated cells from the corresponding groups $(p<0.05)$ and their levels in CL097-treated PBMCs from groups HP, LP, and LP-der were significantly higher than those from mock-infected groups $(p<0.05)$. The TNF- $\alpha$ and IL- 6 concentrations in CL097-stimulated PBMCs from group HP were significantly higher than those from group LP-der $(p<0.05)$. TNF- $\alpha$ secretion in poly(I:C)- and LTA-stimulated, and IL-6 in LTA-stimulated PBMCs from group HP were statistically higher than those from group LP-der $(p<0.05)$. In addition, TNF- $\alpha$ and IL- $1 \beta$ levels in PRRSVtreated cells from groups HP, LP, and LP-der were significantly higher than those in the mock-infected group $(p<0.05)$ (Figure 7).

\section{Discussion}

Pro-inflammatory cytokines, such as IL-1, IL-6, IFN- $\gamma$, and TNF- $\alpha$, are among the first cytokines produced by alveolar macrophages in response to viral infections. The increased circulation levels of these cytokines are responsible for the symptoms of acute systemic inflammation, including fever, depression, and anorexia, which have been associated with cytokine production in lung tissues of PRRSV-infected animals [16,17]. In the present study, HP-PRRSV-infected pigs showed clear clinical signs and high mortality rates and had severe interstitial pneumonia and pneumocytic hyperplasia in their lung tissues. HP virulence was markedly greater than that of LP, which was isolated from clinically ill piglets, and did not have the 30-aa deletion in NSP2 compared to that of PRRSV strain VR-2332. These results were in agreement with earlier in vivo experiments, in which pigs were infected with highly pathogenic American-type PRRSV $[4,18]$. The NT0801-F70 strain derived from NT0801 by propagation in MARC-145 cells to 70 passages showed almost no pathogenicity in piglets. BB0907-infected pigs showed more severe clinical signs and had significantly higher serum levels of TNF- $\alpha$, IL-1 $\beta$, and IL- 6 than those infected with NT0801. Therefore, PRRSV BB0907 and NT0801 isolates could be termed as HP-PRRSV and LP-PRRSV, respectively. IL-1, IL-6, IFN- $\gamma$, and TNF- $\alpha$ expression levels were correlated to PRRSV virulence, which was similar to the results of other reports regarding American serotype 2 HP-PRRSV strain rJXwn06 rescued from an infectious clone of Chinese HP-PRRSV, 

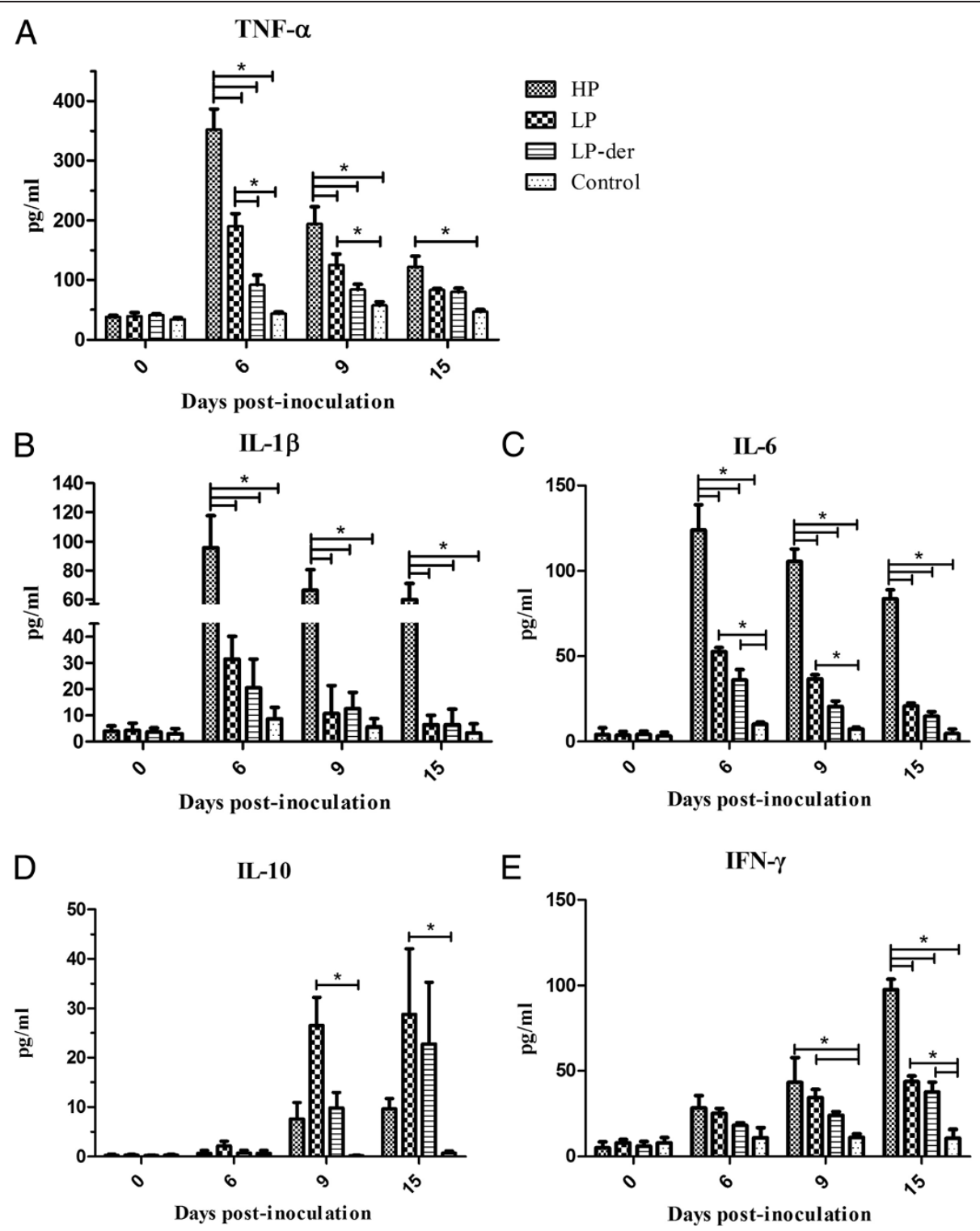

Figure 4 Serum levels of porcine TNF- $\alpha$ (A), IL-1 $\beta$ (B), IL-6 (C), IL-10 (D), and IFN- $\gamma$ (E) were measured with commercial ELISA kits. Data are represented as mean ( \pm SEM) generated from 6 pigs in all groups at 0 to 15 d.p.i. and 4 pigs in group HP at 9 to 15 d.p.i. $\left({ }^{*}\right)$ indicates a significant difference $(p<0.05)$ between groups.

and VR2332 [19], virulent European subtype 3 strain Lena, and low virulent subtype 1 strains, Belgium A and LV [19]. Because the clinical signs in pigs infected with HP- and LP-PRRSV gradually disappeared during the period of 10 to 13 d.p.i, all pigs were euthanized for pathological detection at 15 d.p.i., which was a shorter period than that used in another report [18].

TLRs 3 and 7/8 recognize dsRNA and ssRNA, respectively, and play key roles in viral-mediated innate immune responses. The expression of most TLRs, including TLR3, 7 , and 8, were up-regulated in monocytes of chronic hepatitis C virus-infected patients [19]. Reportedly, TLR3 and 7 mRNA were up-regulated in peripheral and brain tissues of PRRSV-infected pigs. The up-regulation of TLRs, as well as the corresponding neuroinflammatory response, was likely due to the presence of PRRSV in the brain and cytokine-based signals from the peripheral immune system [20]. Infection of pigs with PRRSV tended to upregulate the mRNA expression of TLR2, 3, 4, 7, and 8 in at least one of the lymphoid tissues and PAMs [21]. In contrast, the down-regulation of TLR7 and 8 by poly(I:C) stimulation and PRRSV infection was observed in both PAMs and immature dendritic cells [22]. Interestingly, in the present study, we found that HP infection induced higher expression levels of TLR3, 7, and 8 mRNA in PAMs and cerebral medullar tissues than LP, indicating 


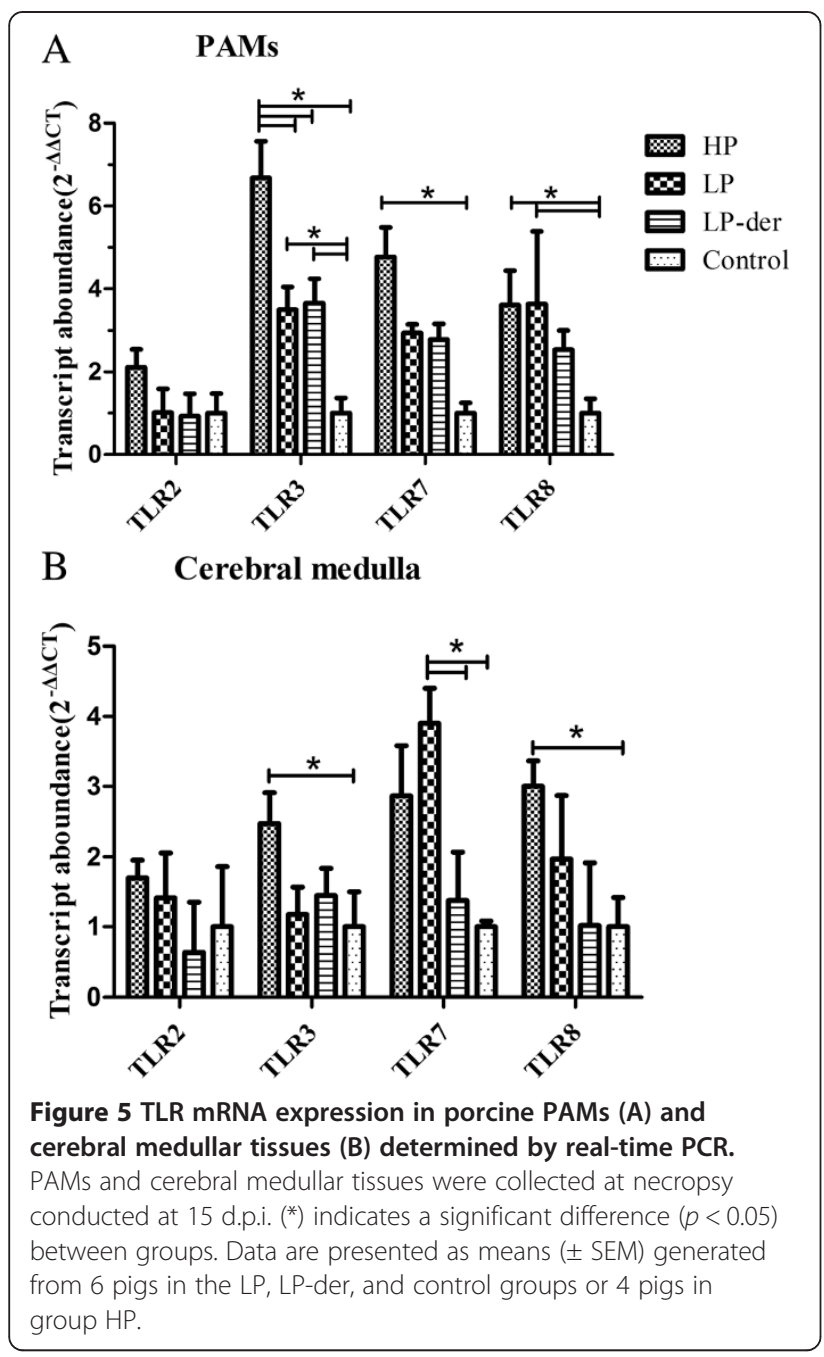

that the TLR expression levels were correlated with PRRSV virulence. Increased TLR expression is an ominous prognostic factor in the host, whereas low TLR expression may protect the host against excessive inflammation and tissue damage. Thus, differential TLR expression in PRRSVinfected pigs may contribute to infection susceptibility and underlying disease progression; however, this supposition remains to be further validated in future studies.

The TLR family can detect distinct microbial PAMPs and trigger the activation of specific signaling pathways leading to the transcription of inflammatory and antiinflammatory cytokines [8]. The exaggerated production of proinflammatory cytokines via the activation of the TLR pathway might contribute to the exacerbated clinical symptoms in combined infections of PRRSV and secondary pathogens. However, the exact mechanisms of interaction between PRRSV and secondary bacterial/viral pathogens remain unclear. Recognition of pathogens requires TLR-mediated signals to initiate innate and subsequent adaptive immune responses. Conceivably, modulated
TLR expression following PRRSV infection in pigs, along with specific PAMPs presented by the secondary pathogens, can significantly influence disease outcome of the combined infection [23]. An in vitro model was established to investigate pro-inflammatory cytokine production by macrophages in response to inoculation with PRRSV and lipopolysaccharides [23]. Synthetic RNA compounds, such as poly(I:C), can activate TLR3-expressing cells [24] and the water-soluble derivative of the imidazoquinoline compound CL097, which acts an a TLR7/8 ligand in the induction of NF- $k B$ activation [25]. In the present study, our results showed that TNF- $\alpha$ levels in poly(I:C)-stimulated PBMCs from group $\mathrm{HP}$ were significantly increased compared to those from the control group. TNF- $\alpha$ and IL- 6 levels in CL097-stimulated PBMCs from groups HP and LP were statistically greater than those from group LP-der. However, the levels of TNF- $\alpha$, IL- $1 \beta$, and IL- 6 in PAMs from group HP were significantly lower than those from group LP. These results might be explained by the low activity of PAMs with high PRRSV load from group HP. Analysis of in vitro PRRSV infectivity in PAMs indicated that pretreatment with the TLR3 ligand, dsRNA, suppressed PRRSV infection (Miller et al., 2009). The authors suggested that TLR3 activation might stimulate protective activity against PRRSV infection. Thus, the up-regulation of TLR3 and TLR7 in lung and brain tissues of PRRSVinfected pigs may restrict viral replication.

IL-10 is an immunomodulatory cytokine that is able to inhibit the synthesis and release of other cytokines [26], thereby inhibiting cell-mediated immunity and extending the duration of viremia in the peripheral blood during the early stage of infection [27]. Although, IL-10 expression was found to be significantly correlated with PRRSV replication [17], it has been reported that some PRRSV strains induce IL-10 secretion in infected macrophages while others do not $[28,29]$. In this study, HP-infected pigs developed low serum IL-10 levels by 15 d.p.i and the level of viremia in the peripheral blood was significantly higher than that in the negative control group. Meanwhile, LPinfected pigs developed higher IL-10 levels compared to those in the other groups. The vFL12 strain, a highly pathogenic PRRSV strain derived from an infectious clone, did not up-regulate IL-10 at the mRNA or protein levels in either infected macrophages or dendritic cells in vitro or in vivo $[28,29]$, suggesting that IL-10 release might not be a part of the PRRSV virulence mechanism.

Usually, HP-PRRSV replicates more efficiently than LP PRRSV and displays an expanded tissue tropism in vivo $[6,30,31]$. For example, infection with the virulent European subtype 3 strain Lena resulted in higher serum viral titers compared to low virulent subtype 1 strains, such as Belgium A and LV [6,30,31]. HP-PRRSV strain HuN4 caused higher viral loads in peripheral blood samples than its derivative strain HuN4-F112 (obtained by propagation in MARC145 

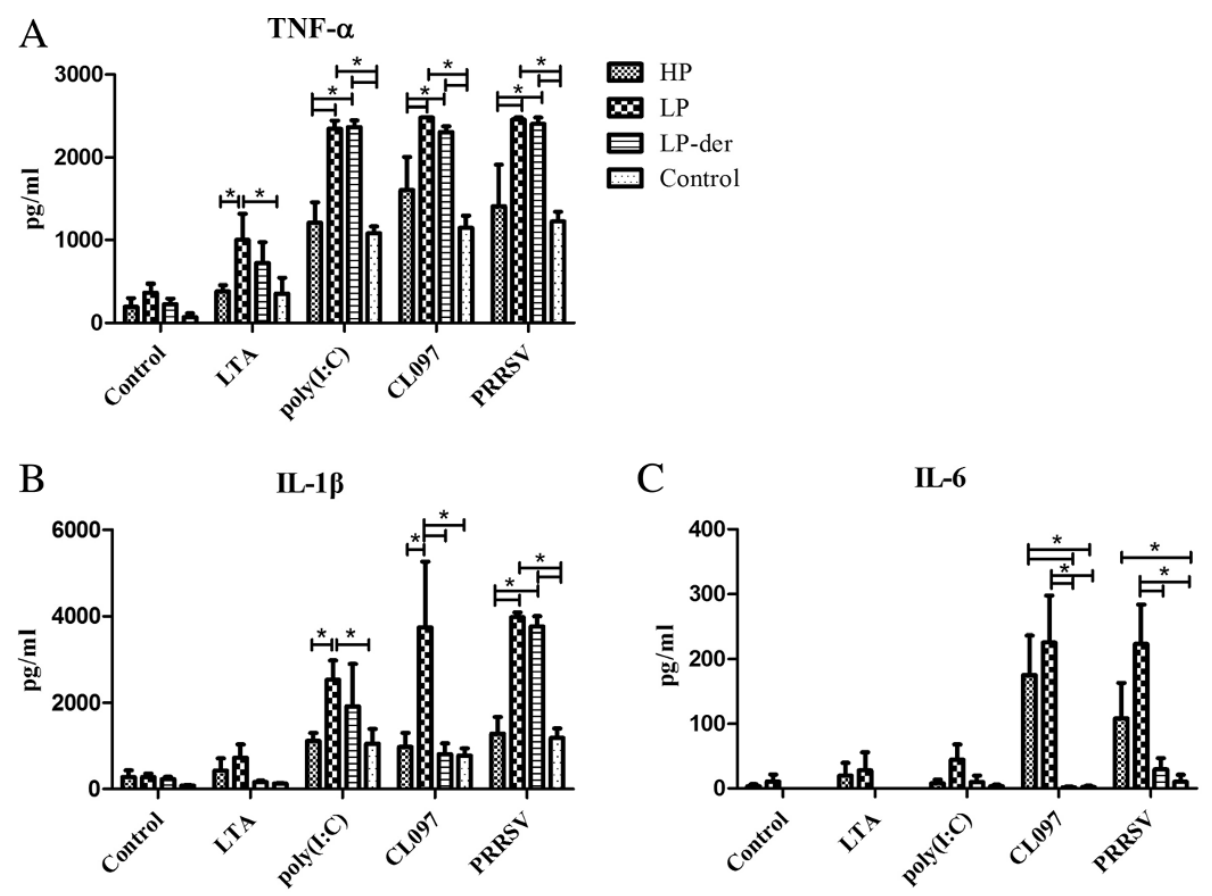

Figure 6 Cytokine production in PAMs following stimulation with LTA, poly(I:C), CL097, or PRRSV from HP, LP, and LP-der PRRSVinfected and mock-infected groups. Cytokine concentrations in the supernatants of stimulated PAMs were determined by ELISA. Data are presented as means $\left( \pm\right.$ SEM) generated from 6 pigs in the LP, LP-der, and control groups or 4 pigs in group HP. $\left(^{*}\right)$ indicates a significant difference between groups $(p<0.05)$.

cells for 112 passages) [6,30,31]. Here, our results showed that LP-PRRSV strain NT0801 induced greater viremia in pigs than its derivative strain NT0801-F70 and those infected with HP-PRRSV strain BB0907 had milder PRRSV viremia than that due to LP-PRRSV strain NT0801, which was isolated from a piglet herd that experienced a morbidity of $\sim 40 \%$ and mortality of $\sim 20 \%$. Strain NT0801 shares a homology of $96.7 \%$ with the HP-PRRSV SY0608 isolate (GenBank accession no.: EU144079), but does not have the 30-aa deletion in NSP2. Furthermore, no obvious recombination signal was observed by comparing its sequence with other PRRSV isolates with different virulence (data not shown). However, the mechanism behind the high viremia level induced by LP-PRRSV strain NT0801 requires further exploration in future studies.

In addition, PRRSV reportedly replicates only in macrophages, but not in monocytes or other peripheral blood cells [32]. However, in the present study, when PBMCs were isolated from pigs infected with the respective PRRSV strain, cultured, and treated with TLR ligands or infected with PRRSV, we found that PRRSV also induced a strong effect on TNF- $\alpha$ and IL- $1 \beta$ secretion, similar to that of the TLR-ligand CL097, although PBMCs are not supposed to contain macrophages. This result may be explained by the maturity of the PBMCs or the activation of the PRRSV antigen protein [32].

\section{Conclusions}

Here, we found that HP was a stronger inducer of TLR $3,7,8$ expression and IL- $1 \beta$, IL- 6 , TNF- $\alpha$, IFN- $\gamma$ production compared to LP and LP-der. Furthermore, HP enhanced production of TNF- $\alpha$, IL-1 $\beta$, and IL- 6 in PBMCs following stimulation with CL097 more so than LP or LP-der, whereas LP enhanced TNF- $\alpha$ and IL-1 $\beta$ secretion in poly(I:C) - and CL097-stimulated PAMs from PRRSV-infected pigs. Together, our results indicate that PRRSV strains with different virulence can differently modulate the immune responses in swine and should be helpful in the development of more efficacious vaccines against PRRSV infection.

\section{Materials and methods \\ Viruses}

A highly pathogenic PRRSV (HP-PRRSV) isolate BB0907 (propagation in MARC-145 cells for 6 passages) (HP) (GenBank no. HQ315835) was isolated in Guangxi Province, China, in 2009. The low pathogenic PRRSV (LP-PRRSV) isolate NT0801 (LP) (GenBank no. HQ315836) was isolated in Jiangsu Province, China, in 2008, and its derivative strain NT0801-F70 (LP-der) was obtained after propagated in MARC-145 cells to 70 passages. The titers of the viral stocks were determined by cytopathic effect (CPE) in MARC-145 

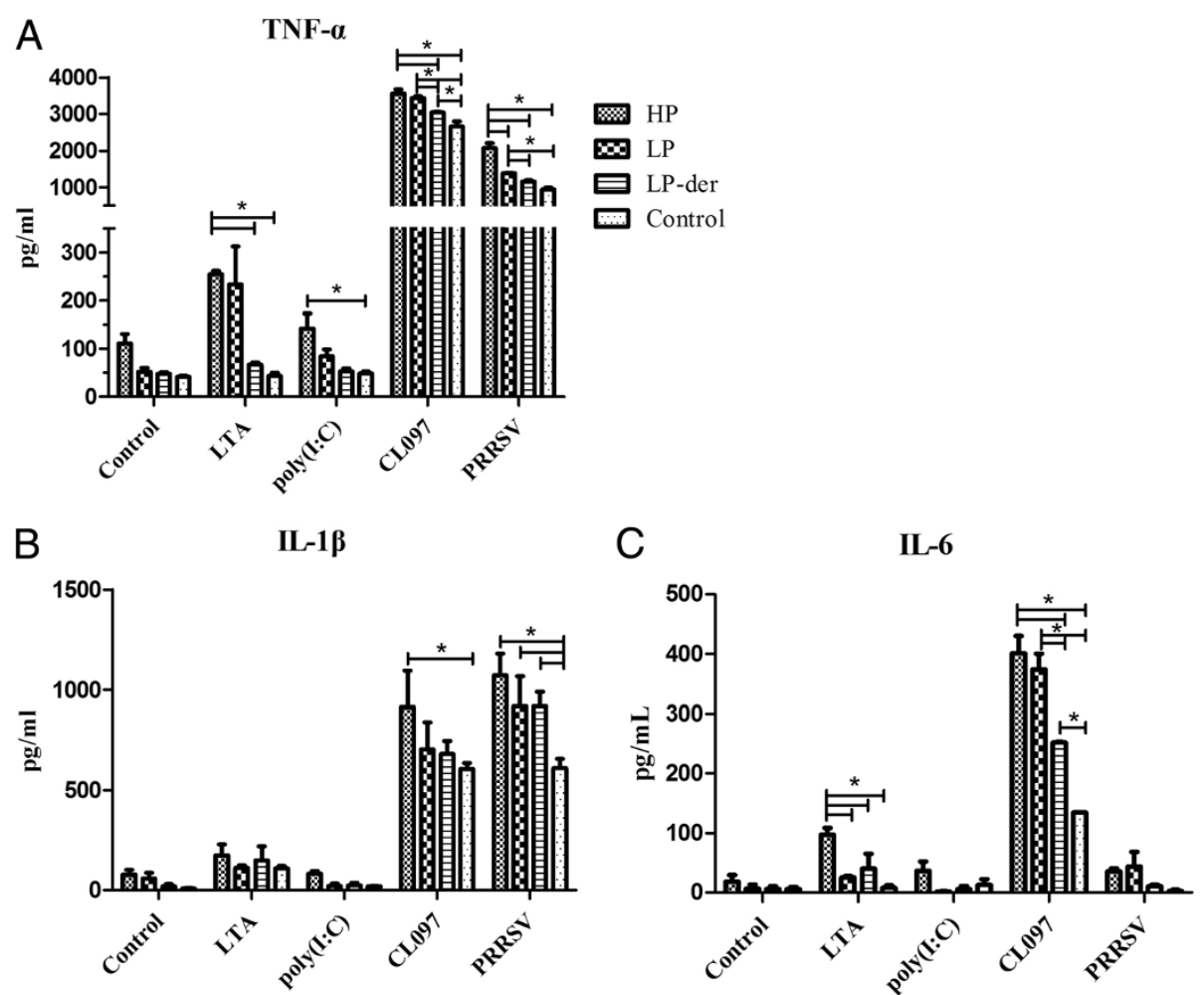

Figure 7 Cytokine production in PBMCs following stimulation with LTA, poly(l:C), CL097, or PRRSV from PRRSV- and mock-infected pigs. Cytokine concentrations in the supernatants of stimulated PBMCs were determined by ELISA. Data are presented as means ( \pm SEM) generated from 6 pigs in the LP, LP-der, and control groups or 4 pigs in group HP. $\left(^{*}\right)$ indicates a significant difference between groups $(p<0.05)$.

cells. The titers of BB0907, NT0801, and NT0801-F70 viral stocks were $10^{5.87}, 10^{6.25}$, and $10^{6.5} \mathrm{TCID}_{50} / \mathrm{ml}$, respectively.

\section{Experimental design}

Twenty-four, 6-week-old, healthy, crossbred piglets free of PRRSV and porcine circovrius type 2 (PCV2) infection were randomly divided into four groups with 6 pigs per group. The pigs in group HP, LP, and LP-der were intranasally inoculated with $2 \mathrm{~mL}$ of the BB0907, NT0801, and NT0801-F70 strains of PRRSV, respectively, with $3 \times 10^{5.5} \mathrm{TCID}_{50} / \mathrm{mL}$ per pig. The pigs in the control group were intranasally inoculated with $2 \mathrm{~mL}$ of Dulbecco modified Eagle's medium (DMEM). The animals were monitored for 15 days post inoculation (d.p. i.). Rectal temperatures and clinical signs were observed daily. At 0, 3, 6, 9, 12, and 15 d.p.i., the blood and sera samples were collected for the detection of PRRSV, IL$1 \beta$, IL-6, IL-10, TNF- $\alpha$, and IFN- $\gamma$. At the end of the experiment, all pigs were euthanized for necropsy. Peripheral blood mononuclear cells (PBMCs), porcine alveolar macrophages (PAMs), and lung and brain tissues were collected for further examination.

This study was approved by the Animal Care and Ethics Committee of Nanjing Agricultural University (permit number: IACECNAU20111105). All authors complied with the "Animal Research: Reporting In Vivo Experiments" (ARRIVE) guidelines [33].

\section{Clinical and pathology examination}

The clinical conditions of the pigs were evaluated daily after challenge as previously reported [34]. Briefly, scores ranged from 1-4, reflecting the severity of the illness, were determined by sum of daily observations of behavior, respiration, and cough. Gross lung lesions were evaluated at necropsy. Gross lesions of each lobe were scored and estimated as the percentage of lung with grossly visible pneumonia, and the overall level of gross lung pathology was determined. Then, the lung tissues were histologically evaluated as previously reported [35].

\section{Preparations of PBMCs and PAMs}

Blood samples from pigs were collected from the jugular veins into blood collection tubes containing heparin lithium before the necropsies. PBMCs were isolated by density gradient centrifugation using lydroxypropylmethyl cellulose (TBDscience, Tianjing, China). Cells were washed three times in phosphate-buffered saline (PBS), counted, and resuspended in RPMI-1640 medium (Gibco- 
Invitrogen, Carlsbad, CA, USA)). PBMCs were dispensed into 24-well-plates (Corning, Inc., Corning, NY, USA) at $10^{6}$ cells/well in a volume of $500 \mu \mathrm{L}$. The cells were stained and subjected to differential counting based on cell morphology. For PBMC cultures, both adherent and non-adherent cells were used for the study.

The PAMs were isolated from bronchoalveolar lavage by centrifugation as described previously [21]. PAMs were diluted to $2 \times 10^{6}$ cells $/ \mathrm{mL}$ and dispensed into 24-well plates (Corning, Inc.) or $25-\mathrm{cm}^{2}$ flasks (Corning, Inc.) in a volume of $500 \mu \mathrm{L}$ or $5 \mathrm{~mL}$. The differential counting was performed on stained cytospin slides of the PAMs. Nonadherent cells were removed by gentle aspiration of the culture supernatant and replaced with fresh media.

\section{Stimulation of PAMs and PBMCs with PRRSV or microbial PAMPs}

PAMs and PBMCs dispensed into 24-well plates were treated with LTA from Staphylococcus aureus $(10 \mu \mathrm{g} / \mathrm{mL}$; InvivoGen, San Diego, CA, USA), poly(I:C) $(10 \mu \mathrm{g} / \mathrm{mL}$; InvivoGen), CL097 (5 $\mu \mathrm{g} / \mathrm{mL}$; InvivoGen), PRRSV (BB0907, NT0801, NT0801-F70, 0.1MOI), or they remained untreated (control). Stimulated PAMs and PBMCs were then cultured in a $5 \% \mathrm{CO}_{2}$ incubator at $37^{\circ} \mathrm{C}$ for $48 \mathrm{~h}$. Supernatants were then collected from the cell cultures for cytokine concentration determination by ELISA. To investigate TLR mRNA expression, PAMs in $25-\mathrm{cm}^{2}$ flasks obtained from PRRSV- and mock-infected pigs were used for RNA extraction.

\section{Cytokine measurement}

TNF- $\alpha$, IL-1 $\beta$, IL-6, IL-10, and IFN- $\gamma$ were measured in pig serum or the culture supernatants using porcine specific ELISA kits (R\&D Systems, Minneapolis, MN, USA) according to the manufacture's instructions. A standard curve was generated using known concnetrations. All detections were performed in parallel.

\section{Real-time PCR for detection of PRRSV in serum}

Total RNA was extracted from serum using TRIzol reagent (Invitrogen, Carlsbad, CA, USA) according to the manufacturer's instructions. Reverse transcription was performed using M-MLV Reverse Transcriptase (Promega, Madison, WI, USA) in a $25-\mu \mathrm{L}$ reaction mixture containing $2 \mu \mathrm{g}$ of RNA according to the manufacturer's instructions. Two microliters of the RT reaction mixture was subjected to real-time PCR analysis using ORF7-specific primers (sense: 5'-AAT AAC AAC GGC AAG CAG CAG-3'; antisense: 5'-CCT CTG GAC TGG TTT TGC TGA-3'), and SYBR Green Real-time PCR Master Mix (Toyobo Co., Ltd., Osaka, Japan), according to the Manufacturer's recommendations. The reaction procedure included one a denaturing stage at $95^{\circ} \mathrm{C}$ for $2 \mathrm{~min}$, followed by 40 cycles at $95^{\circ} \mathrm{C}$ for $15 \mathrm{~s}$ and $60^{\circ} \mathrm{C}$ for $1 \mathrm{~min}$. To confirm specific amplification, melting curve analysis of the RT-PCR products was performed according to the manufacturer's protocol. Realtime PCR was performed in an ABI PRISM 7300 sequence detection system and analyzed with ABI PRISM $7300_{\text {SDS }}$ software (Applied Biosystems, Foster City, CA, USA). For each assay a standard curve was generated using serially diluted PRRSV as described previously [36]. Slope $=-3.551481$; $\mathrm{R}^{2}=0.988176$; PCR efficiency $=0.9123$.

\section{Real-time PCR for detection of TLR mRNA}

Total RNA was extracted from PAMs and brain tissues as described above. Based on the sequence of porcine TLR2, TLR3, TLR7, TLR8, $\beta$-actin, and $18 \mathrm{~S}$ ribosomal RNA (18S rRNA) (GenBank number in Table 1), the probes and primers were designed using Primer Express version 3.0 software (Applied Biosystems) as shown in Table 2. All

Table 2 Sequences of porcine-specific real-time RT-PCR primers and TaqMan probes

\begin{tabular}{|c|c|c|c|}
\hline Genes $^{a}$ & Primer sequence $\left(5^{\prime}-3^{\prime}\right)^{\mathbf{b}}$ & Probe sequence $\left(5^{\prime}-3^{\prime}\right)^{c}$ & Accession number $^{d}$ \\
\hline \multirow[t]{2}{*}{ TLR2 } & F:TGCTGGAACCCATCGAGAA & AAGGCCATTCCCCAGCGTTTCTGTAA & GU138028 \\
\hline & R:AGGTAGGTCCTGGTGTTCATTATCTT & & \\
\hline \multirow[t]{2}{*}{ TLR3 } & F:CCCAGTGATTCTIITTGATACATCA & CCTGCAAAGACAGTGCCCCATTTGA & GU013759 \\
\hline & R:CATACTGGCACTTATCATGAAAAAGAG & & \\
\hline \multirow[t]{2}{*}{ TLR7 } & F:CCAACAACCGGCTTGATTTAC & CAACAGCATTCGAAGAGCTACGCAACC & GU013760 \\
\hline & R:TCTGATTGAAAATAGTGGCTGTTACTACT & & \\
\hline \multirow[t]{2}{*}{ TLR8 } & F: AAAAAGCACGTCCCTGAAAGAA & TTTCAGTGGAAACCGCCTGGACCTT & GU013761 \\
\hline & R:TACCTGTCATCTTGGGCATTCC & & \\
\hline \multirow[t]{2}{*}{$\beta$-actin } & F:TCTTCCAGCCCTCCTTCCT & AGTCCTGCGGCATCCACGAGACC & U07786 \\
\hline & R:ACGTCGCACTTCATGATCGA & & \\
\hline \multirow[t]{2}{*}{$18 \mathrm{~S}$ rRNA } & F:CCCCAACTTCTTAGAGGGACAA & TGGCGTTCAGCCACCCGAGATTT & DQ437859 \\
\hline & R:GGGCATCACAGACCTGTTATTG & & \\
\hline
\end{tabular}

${ }^{a}$ TLR2, Toll-like receptor 2; TLR3, Toll-like receptor 3; TLR7, Toll-like receptor 7; TLR8, Toll-like receptor 8; $18 \mathrm{~S}$ rRNA, $18 \mathrm{~S}$ ribosomal RNA.

${ }^{\mathrm{b}} \mathrm{F}$, forward primer; $\mathrm{R}$, reverse primer.

CAll probes contained FAM as a fluorescent reporter dye at 5'end and TAMRA as quencher dye at the 3'end. 
Taqman minor groove binding oligonucleotide probes contained FAM as a fluorescent reporter dye at the 5' end and TAMRA as a quencher dye at the 3 ' end. Appropriate reference genes for real-time RT-PCR were selected based on preliminary analyses indicating similar expression of the control genes among the four treatment groups. $\beta$ actin (PAMs) and 18S rRNA (brain) were used as reference genes for data normalization. The levels of TLRs mRNA from cDNA in the samples were calculated using the following formula: $2^{-\Delta \Delta \mathrm{Ct}}=2^{\Delta \mathrm{Ct} \text { TLRs }} / 2^{\Delta \mathrm{Ct} \beta \text {-actin }} \cdot \Delta \mathrm{Ct}$ TLRs $=$ Ct TLRs of the cells at indicated time-Ct TLRs of the cells at corresponding time; $\Delta \mathrm{Ct} \beta$-actin $=\mathrm{Ct} \beta$-actin of the cells at corresponding time -Ct $\beta$-actin of the cells at corresponding time [20].

The total PCR reaction volume was $20 \mu \mathrm{L}$, including 2 $\mu \mathrm{L}$ of cDNA templates, $10 \mu \mathrm{M}$ of each primer and probe, $2 \times$ Premix Ex Taq (Takara Co., Ltd., Shinga, Japan). The PCR conditions were as follows: predenaturing at $95^{\circ} \mathrm{C}$ for $30 \mathrm{~s}$ and 45 cycles of denaturing at $95^{\circ} \mathrm{C}$ for $15 \mathrm{~s}$, annealing at $56^{\circ} \mathrm{C}$ for $15 \mathrm{~s}$, and an extension at $72^{\circ} \mathrm{C}$ for $31 \mathrm{~s}$. The information of the real-time PCR results was shown in Additional file 1: Figure S1. Those PCR efficiencies were $0.91068 \sim 1.08137$.

\section{Statistical analysis}

Results were analyzed for significance with the two-way analysis of variance test using GraphPad PRISM software (version 5.02 for Windows; www.graphpad.com/). A $p$ value $<0.05$ was considered significantly significant.

\section{Additional file}

Additional file 1: Figure S1. Standard curves for quantitation of TLRS, $\beta$-actin and 18S rRNA by TaqMan Real-time PCR. (A) Amplification curves obtained with serial dilutions of TLRs, $\beta$-actin and $18 \mathrm{~S}$ rRNA genes, The $x$-axis shows the number of PCR cycles and the $y$-axis shows the normalized fluorescence intensity (Rn). (B) The standard curves of TLRs, $\beta$-actin and $18 \mathrm{~S}$ rRNA genes show comparable slopes, indicating similar PCR efficiency

\section{Abbreviations}

HP: Highly pathogenic PRRSV strain BB0907; HP-PRRSV: Highly pathogenic PRRSV; LP: Low pathogenic PRRSV strain NT0801; LP-der: Low pathogenic PRRSV derivative strain NT0801-F70; LP-PRRSV: Low pathogenic PRRSV; PAMs: Porcine alveolar macrophages; PBMCs: Peripheral blood mononuclear cells; PRRSV: Porcine reproductive and respiratory syndrome virus; TLRs: Tolllike receptors.

\section{Competing interests}

The authors declare that they have no competing interests.

\section{Author contributions}

LZ performed most of the experiments and wrote the manuscript. PJ critically revised the manuscript and the experimental design. JL collected samples and gathered clinical data. JB and XW analyzed the animal experimental data and edited the draft manuscript. YL analyzed the histopathological data. All authors read and approved the final version of the manuscript.

\section{Acknowledgements}

The work was mainly supported by the national natural science foundation (31230071) and grants from the Ministry of Education, China (313031, 20120097110043) for PRRSV immunology, a grant from the ministry of agriculture (CARS-36) for swine disease control techniques, and priority academic program development of the Jiangsu higher education institution (PAPD).

Received: 24 July 2012 Accepted: 18 April 2013

Published: 30 April 2013

\section{References}

1. Meulenberg JJ, Hulst MM, de Meijer EJ, Moonen PL, den Besten A, de Kluyver EP, Wensvoort G, Moormann RJ: Lelystad virus, the causative agent of porcine epidemic abortion and respiratory syndrome (PEARS), is related to LDV and EAV. Virology 1993, 192:62-72.

2. Christianson WT, Collins JE, Benfield DA, Harris L, Gorcyca DE, Chladek DW, Morrison RB, Joo HS: Experimental reproduction of swine infertility and respiratory syndrome in pregnant sows. Am J Vet Res 1992, 53:485-488,

3. Rossow KD, Bautista EM, Goyal SM, Molitor TW, Murtaugh MP, Morrison RB, Benfield DA, Collins JE: Experimental porcine reproductive and respiratory syndrome virus infection in one-, four-, and 10-week-old pigs. I Vet Diagn Invest 1994, 6:3-12.

4. Li Y, Wang X, Bo K, Tang B, Yang B, Jiang W, Jiang P: Emergence of a highly pathogenic porcine reproductive and respiratory syndrome virus in the Mid-Eastern region of China. Vet J 2007, 174:577-584.

5. Tian K, Yu X, Zhao T, Feng Y, Cao Z, Wang C, Hu Y, Chen X, Hu D, Tian X, et al: Emergence of fatal PRRSV variants: unparalleled outbreaks of atypical PRRS in China and molecular dissection of the unique hallmark. PLoS One 2007, 2:e526.

6. Zhou YJ, Hao XF, Tian ZJ, Tong GZ, Yoo D, An TQ, Zhou T, Li GX, Qiu HJ, Wei TC, Yuan XF: Highly virulent porcine reproductive and respiratory syndrome virus emerged in China. Transbound Emerg Dis 2008, 55:152-164.

7. Gale M Jr, Foy EM: Evasion of intracellular host defence by hepatitis $C$ virus. Nature 2005, 436:939-945.

8. Krishnan J, Selvarajoo K, Tsuchiya M, Lee G, Choi S: Toll-like receptor signal transduction. Exp Mol Med 2007, 39:421-438.

9. Matsumoto M, Funami K, Oshiumi H, Seya T: Toll-like receptor 3: a link between toll-like receptor, interferon and viruses. Microbiol Immunol 2004, 48:147-154.

10. Schulz O, Diebold SS, Chen M, Naslund TI, Nolte MA, Alexopoulou L, Azuma YT, Flavell RA, Liljestrom P, Reis e Sousa C: Toll-like receptor 3 promotes cross-priming to virus-infected cells. Nature 2005, 433:887-892.

11. Yamamoto M, Sato S, Mori K, Hoshino K, Takeuchi O, Takeda K, Akira S: Cutting edge: a novel Toll/IL-1 receptor domain-containing adapter that preferentially activates the IFN-beta promoter in the Toll-like receptor signaling. J Immunol 2002, 169:6668-6672.

12. Akira S, Uematsu S, Takeuchi O: Pathogen recognition and innate immunity. Cell 2006, 124:783-801

13. Li Y, Wang X, Jiang P, Chen W: Genetic analysis of two porcine reproductive and respiratory syndrome viruses with different virulence isolated in China. Arch Virol 2008, 153:1877-1884.

14. Wang C, Wu B, Amer S, Luo J, Zhang H, Guo Y, Dong G, Zhao B, He H: Phylogenetic analysis and molecular characteristics of seven variant Chinese field isolates of PRRSV. BMC Microbiol 2010, 10:146.

15. Zhou L, Chen S, Zhang J, Zeng J, Guo X, Ge X, Zhang D, Yang H: Molecular variation analysis of porcine reproductive and respiratory syndrome virus in China. Virus Res 2009, 145:97-105.

16. Thanawongnuwech R, Thacker B, Halbur P, Thacker EL: Increased production of proinflammatory cytokines following infection with porcine reproductive and respiratory syndrome virus and Mycoplasma hyopneumoniae. Clin Diagn Lab Immunol 2004, 11:901-908.

17. Gomez-Laguna J, Salguero FJ, Barranco I, Pallares FJ, Rodriguez-Gomez IM, Bernabe A, Carrasco L: Cytokine expression by macrophages in the lung of pigs infected with the porcine reproductive and respiratory syndrome virus. J Comp Pathol 2010, 142:51-60.

18. Liu Y, Shi W, Zhou E, Wang S, Hu S, Cai X, Rong F, Wu J, Xu M, Li L: Dynamic changes in inflammatory cytokines in pigs infected with highly pathogenic porcine reproductive and respiratory syndrome virus. Clin Vaccine Immunol 2010, 17:1439-1445. 

expression in monocytes and T cells in chronic HCV infection. World J Gastroenterol 2006, 12:1198-1204

20. Miguel JC, Chen J, Van Alstine WG, Johnson RW: Expression of inflammatory cytokines and Toll-like receptors in the brain and respiratory tract of pigs infected with porcine reproductive and respiratory syndrome virus. Vet Immunol Immunopathol 2010, 135:314-319.

21. Liu CH, Chaung HC, Chang HL, Peng YT, Chung WB: Expression of Toll-like receptor mRNA and cytokines in pigs infected with porcine reproductive and respiratory syndrome virus. Vet Microbiol 2009, 136:266-276.

22. Chaung HC, Chen CW, Hsieh BL, Chung WB: Toll-Like Receptor expressions in porcine alveolar macrophages and Dendritic Cells in responding to poly IC stimulation and porcine reproductive and respiratory syndrome virus (PRRSV) infection. Comp Immunol Microbiol Infect Dis 2010, 33:197-213.

23. Qiao S, Feng L, Bao D, Guo J, Wan B, Xiao Z, Yang S, Zhang G: Porcine reproductive and respiratory syndrome virus and bacterial endotoxin act in synergy to amplify the inflammatory response of infected macrophages. Vet Microbiol 2011, 149:213-220.

24. Takeuchi $\mathrm{O}$, Hemmi H, Akira S: Interferon response induced by Toll-like receptor signaling. J Endotoxin Res 2004, 10:252-256.

25. Miller LC, Lager KM, Kehrli ME Jr: Role of Toll-like receptors in activation of porcine alveolar macrophages by porcine reproductive and respiratory syndrome virus. Clin Vaccine Immunol 2009, 16:360-365.

26. Moore KW, de Waal MR, Coffman RL, O'Garra A: Interleukin-10 and the interleukin-10 receptor. Annu Rev Immunol 2001, 19:683-765.

27. Wang G, Song T, Yu Y, Liu Y, Shi W, Wang S, Rong F, Dong J, Liu H, Cai X, Zhou EM: Immune responses in piglets infected with highly pathogenic porcine reproductive and respiratory syndrome virus. Vet Immunol Immunopathol 2011, 142:170-178.

28. Subramaniam S, Sur JH, Kwon B, Pattnaik AK, Osorio FA: A virulent strain of porcine reproductive and respiratory syndrome virus does not up-regulate interleukin-10 levels in vitro or in vivo. Virus Res 2011, 155:415-422

29. Diaz I, Darwich L, Pappaterra G, Pujols J, Mateu E: Different European-type vaccines against porcine reproductive and respiratory syndrome virus have different immunological properties and confer different protection to pigs. Virology 2006, 351:249-259.

30. Tian K, Yu X, Zhao T, Feng Y, Cao Z, Wang C, Hu Y, Chen X, Hu D, Tian X Emergence of fatal PRRSV variants: unparalleled outbreaks of atypical PRRS in China and molecular dissection of the unique hallmark. PLOS One 2007, 2

31. Li Y, Wang $X$, Bo K, Wang X, Tang B, Yang B, Jiang W, Jiang P: Emergence of a highly pathogenic porcine reproductive and respiratory syndrome virus in the Mid-Eastern region of China. Vet J 2007, 174:577-584.

32. Rutherford MS, Witsell A, Schook LB: Mechanisms generating functionally heterogeneous macrophages: chaos revisited. J Leukoc Biol 1993, 53:602-618

33. Kilkenny C, Browne W, Cuthill IC, Emerson M, Altman DG: Animal research reporting in vivo experiments: the ARRIVE guidelines. Br J Pharmacol 2010, 160:1577-1579.

34. Wang Y, Liang Y, Han J, Burkhart KM, Vaughn EM, Roof MB, Faaberg KS: Attenuation of porcine reproductive and respiratory syndrome virus strain MN184 using chimeric construction with vaccine sequence. Virology 2008, 371:418-429.

35. Halbur PG, Miller LD, Paul PS, Meng XJ, Huffman EL, Andrews JJ: Immunohistochemical identification of porcine reproductive and respiratory syndrome virus (PRRSV) antigen in the heart and lymphoid system of three-week-old colostrum-deprived pigs. Vet Pathol 1995, 32:200-204.

36. Li G, Jiang P, Li Y, Wang X, Huang J, Bai J, Cao J, Wu B, Chen N, Zeshan B: Inhibition of porcine reproductive and respiratory syndrome virus replication by adenovirus-mediated RNA interference both in porcine alveolar macrophages and swine. Antiviral Res 2009, 82:157-165.

doi:10.1186/1743-422X-10-135

Cite this article as: Zhang et al:: Comparative expression of Toll-like receptors and inflammatory cytokines in pigs infected with different virulent porcine reproductive and respiratory syndrome virus isolates. Virology Journal 2013 10:135.

\section{Submit your next manuscript to BioMed Central and take full advantage of:}

- Convenient online submission

- Thorough peer review

- No space constraints or color figure charges

- Immediate publication on acceptance

- Inclusion in PubMed, CAS, Scopus and Google Scholar

- Research which is freely available for redistribution 